\title{
DOA MEASUREMENTS ON INDOOR CHANNEL BASED ON MUSIC AND MDL PROCESSING
}

\author{
Yasar Kurdi and Leandro de Haro \\ (1) Dpto. Señales, Sistema y Radiocomunicaciones Universidad Politécnica de Madrid \\ E.T.S. de Ing. de Telecomunicación. Ciudad universitaria 28040 Madrid (SPAIN) \\ vasar@gr.ssr.upm.es, leandro@gr.ssr.upm.es
}

\begin{abstract}
Introduction
In indoor communications, the multipath propagation is fundamental. Generally, several replicas of the signal impinge in the receiving antenna. When there is Line Of Sight, the received energy will be centered in a single wave. In the opposite case, the energy received in the Array, is divided in several directions of the multipath, the signal simulates to have arrived from several sources of signal. In our case, using the results of a measurement campaign of the radio channel frequency response in an indoor scattered environment, we esteem the Direction Of Arrival (DOA) of our signal, using MUSIC algorithm (MUltiple SIgnal Classification) associated with Smoothing Pre-processing and using MDL (Minimum Description Length) approach proposed by Wax and Kailath for the detection of the number of multipath rays arrived at the antenna.
\end{abstract}

\section{Measurement Procedure}

The measurement has been done for different positions between the transmitting and receiving antennas. It was carried out by fixing the transmitting antenna and moving the receiving antenna on a track with displacement interval of $1 / 8$ of the wavelength $(15.5 \mathrm{~mm})$. The receiving antennas on the axes $\mathrm{X}, \mathrm{Y}$ and $\mathrm{Z}$, that is with a 90 angle of degree among them.

A Vector network analyzer HP8510C has been used in the measurements. The signal generator operates in the frequency range between $10 \mathrm{MHz}$ and $50 \mathrm{GHz}$, in our case, to the band of study is between $2,3 \mathrm{GHz}$ and $2,5 \mathrm{GHz}$ with a bandwidth of $200 \mathrm{MHz}$ and central frequency of $2,4 \mathrm{GHz}$.

The transmission and reception antennas are of the same type and correspond to the wide band omni-directional antenna EM-6865 (made by Electro-Metrics) with vertical polarization and typical gain of $0 \mathrm{~dB}$. In the measurement band this antenna has a factor of $40,3 \mathrm{~dB} / \mathrm{m}$.[1]

\section{Description of the environments}

The transmitting antenna (vertically polarized) is placed fixed to the ceiling of an electronic components laboratory (the antenna is at $220 \mathrm{~cm}$ ), in this laboratory we found several electronic equipment (oscilloscopes, etc) placed on tables of $150 \mathrm{~cm}$ height, in addition to several metallic closets among other obstacles. [2]

In a contiguous laboratory separated by a brick wall, two measurements series have been done. Near the mentioned wall, a metallic panel and the equipment of measurement (HP8510C) and the table of control are there. In the laboratory, there are several tables with computers and other electronic equipment (see the details in the plane (figure1)). 
These two series of measurements have been done in a crossed form where the reception antenna has a non line of sight with the transmitting one.

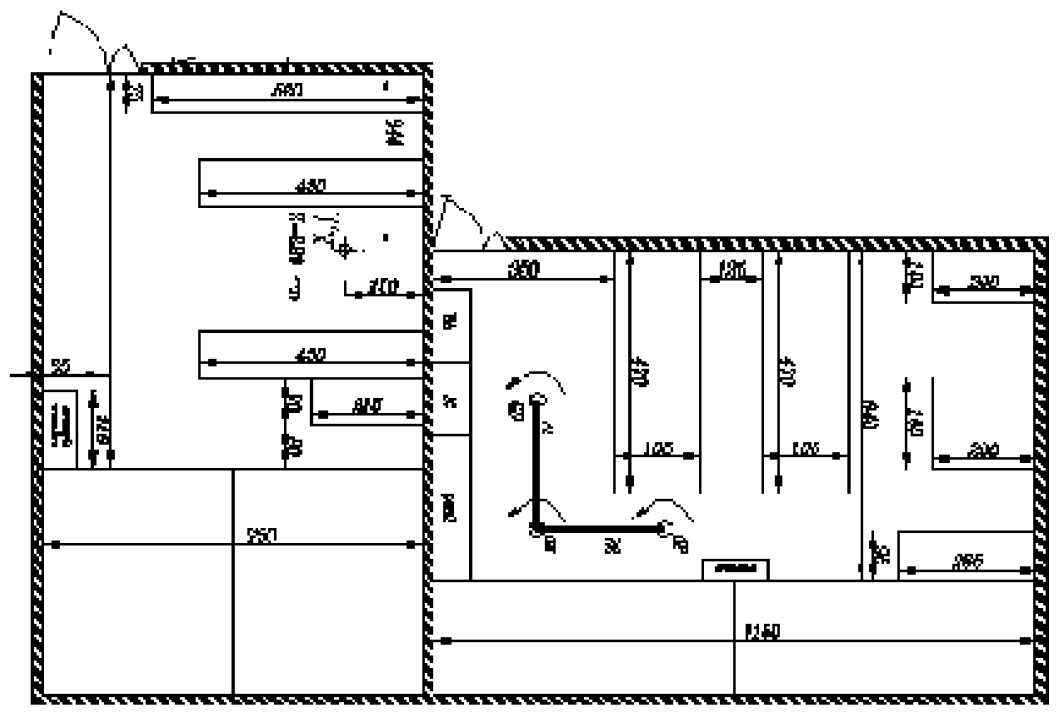

Fig. (1). Measurement site plane

\section{Description and problem formulation:}

The measurement has been performed in an indoor environment using a vector network analyzer (HP8510) on the 2.3 to $2.5 \mathrm{GHz}$ frequency band. The three receiving antennas were placed in a computer laboratory following virtually the three Cartesian axis $\mathrm{X}, \mathrm{Y}$, $Z$, The receiving antenna, was formed by a linear Array of 42 (from 168 set of measurements) equal antennas separated of one eighth of a wavelength $(15.5 \mathrm{~cm})$. The transmitting antenna was hanged from the ceiling at the room beside.

For that reason, we have an uniform linear Array of $\mathrm{N}$ sensors, on each one $\mathrm{M}$ plan waves impinge from directions $\theta 1, \ldots, \theta \mathrm{M}$, the received signal can be expressed by

$$
y(t)=\sum_{m=1}^{M} a\left(\theta_{m}\right) S_{m}(t)+w(t)
$$

Where $S(t)$ is the vector of the signal, w(t) is the vector noise and $a(\theta)$ is the Array response or the steering vector. The MUSIC Algorithm proposed by Schmidt [3] is based on the orthogonality between the signal and the noise subspaces. The power spectrums of MUSIC, which are a function of $\theta$, given by:

$$
P_{M U}(\theta)=\frac{1}{a^{H}(\theta) E_{n} E_{n}^{H} a(\theta)}
$$

The correlations between the arrival signals should be suppressed. In order to "decorrelate" the signals, we employ the spatial smoothing pre-processing (SSP) method 
[4] before using the MUSIC algorithm. the main sensor array is divided into L sub-arrays with the same properties. Covariance matrixes of the sub-arrays are then calculated and their average is used as the modified array covariance matrix. In order to de-correlate $\mathrm{N}$ coherent signals, at least $\mathrm{N}$ sub-arrays are required and the spatially smoothed covariance matrix is

$$
R_{S S P}=\frac{1}{M} \sum_{i=1}^{M} R_{i}
$$

where $\mathbf{M}(M \succ N)$, is the number of sub-arrays. $\mathrm{R}_{\mathrm{i}}$ is the covariance matrix of the $\mathrm{M}$-th sub-array.

The peaks of this function occur when the Steering vector and the noise subspace are orthogonal. In Scattered environment exist an important and critical difficult in the estimation of the Direction Of Arrival, that, the detection of the sources number that means the division of the received signal in subspace of signal and noise, Wax and Kailath [5] proposed, MDL method, where the number of signals is obtained by minimizing the MDL function over $\theta(\mathrm{k})$

$$
M D L(k)=-2 \log \left(\frac{\prod_{i=k+1}^{p} l_{i=k+1}^{1 /(p-k)}}{\frac{1}{p-k} \sum_{i=k+1}^{p} l_{i}}\right)^{(p-k) N}+\frac{1}{2} k(2 p-k) \log N
$$

Where $\mathrm{p}$ is the number of antennas $\mathrm{l}_{\mathrm{i}}$ are the eigenvalues of $\mathrm{R}=\psi+\sigma^{2} \mathrm{I}$ and $\psi=\mathrm{aSa}^{\mathrm{T}}$.

\section{Experimental Results}

Figure (2) and Figure (3) show the different Directions Of Arrival for all Cartesians axis (X, Y and $\mathrm{Z}$ ) in a scattered indoor environment for the two orthogonal sets of measurement, we denote the change on Power Spectrum of Doa in each set of measurement caused by the presence of several reflecting objects in the environment. We can see clearly also, the changes of the Direction Of Arrival with the change of the polarization, also we can denote the Power Spectrum depending of the reception polarization, which will be very useful for use the diversity by polarization in MIMO system. 

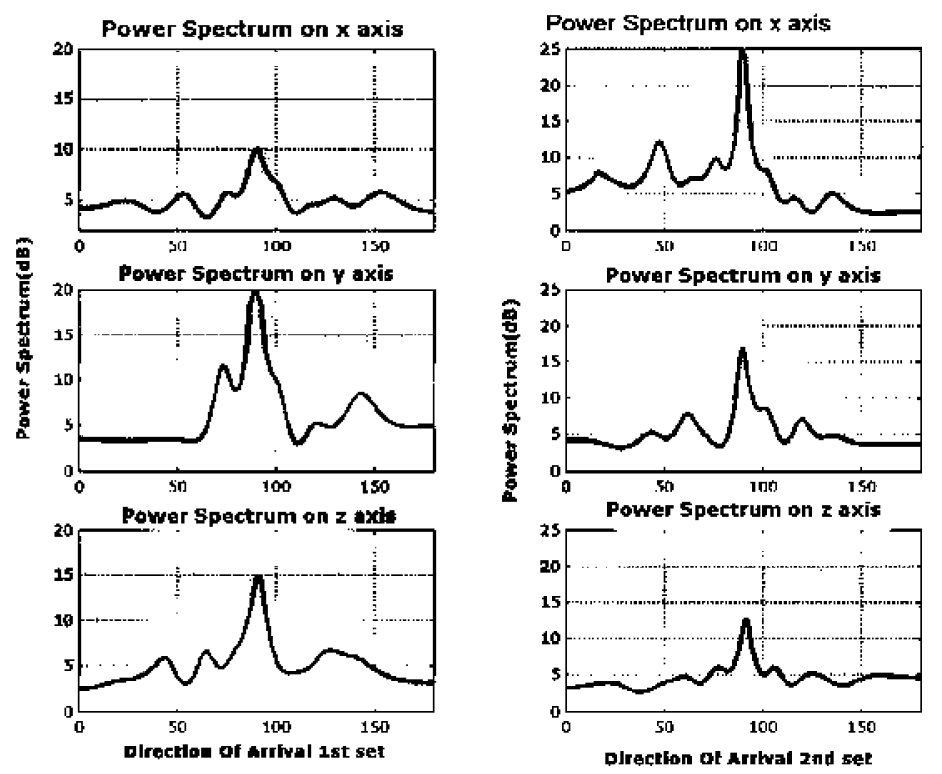

Fig.(2) DOA results for $1^{\text {st }}$ set track Fig. (3) DOA results for $2^{\text {nd }}$ set track.

\section{References:}

[1] Omni-Directional Wideband Antenna, Model EM-6865, 2GHz - 18GHz, Instruction Manual, Electro-Metric, Inc, 1997.

[2] Kurdi, Y.; de Haro y Ariel, L.; Sanchez, M.G, DOA for polarization components and diversity combination from indoor measurements Antennas and Propagation Society International Symposium, 2005 IEEE Volume 4A, 3-8 July 2005 Page(s): 142 - 145 vol. 4A

[3] R. O. Schmidt, Multiple Emitter Location and Signal Parameter Estimation, IEEE Transactions on Antennas and Propagation, vol. -AP34, No 3, March 1986.

[4] T. J. Shan, M. Wax, and T. Kailath, "On spatial smoothing for direction- of-arrival estimation of coherent signals," IEEE Trans. Acoust., Speech, Signal Processing, vol. ASSP-33, pp. 806-811, Aug. 1985.

[5] M. Wax and T. Kailath, Detection of signals by information Theoretic Criteria, IEEE Transactions on Acoustics, Speech, and Signal Processing, Vol. ASSP-33, N ${ }^{\circ}$. 2,April 1985 